\title{
The Low-Level Cognitive Processes Involved in the Visual Search of Pull-Down Menus and Computer Screens, as Revealed by Cognitive Modeling
}

\author{
Anthony J. Hornof \\ Artificial Intelligence Laboratory \\ Electrical Engineering \& Computer Science Department \\ University of Michigan \\ 1101 Beal Avenue, Ann Arbor, MI 48109-2110 \\ +13137631563 \\ hornof@umich.edu
}

\begin{abstract}
The field of human-computer interaction does not have an empirically validated model of the low-level perception, decision, and motor processes that people use when they look for an item on a computer screen. The goal of this dissertation is to provide such a model. The approach will be to (1) build computational cognitive models that simulate people accomplishing various specific visual search tasks, such as menu selection tasks, (2) validate the proposed visual search models by using the models to accurately predict how much time people require to accomplish the same visual search tasks, and (3) provide the field of human-computer interaction with some specific practical benefit from these models, such as explanations of how screen layout design guidelines affect the cognitive processes involved in visual search.
\end{abstract}

\section{Keywords}

Cognitive models, menu selection, visual search.

\section{INTRODUCTION}

A major challenge in making software easy for people to use is to design screen layouts that people can search efficiently. Although there has been a great deal of research on visual search, the field of human-computer interaction still does not have an empirically validated model of the low-level perception, decision, and motor processes that people use when they look for an item on a computer screen. As well, there are many guidelines to direct the design of computer screen layouts, but few if any of these guidelines have been explained in terms of how they affect the low level cognitive processes involved in visual search tasks. Nevertheless, modeling that Hornof \& Kieras have accomplished thus far (discussed in [2]) suggests that a lowlevel model predicting aspects of visual search is feasible at least in the domain of menu search, and probably as well in the domain of general search of computer screen layouts.

\section{PREVIOUS RESEARCH}

Previous researchers have studied visual search of menus and computer screen layouts. They have collected observational data, proposed theories, and offered guidelines

CCopyright on this material is held by the author. for designing menus and interfaces that can be searched efficiently. Previous researchers have also written computer programs that predict search times [9], that automatically evaluate the efficiency of screen layouts [8], and that model graphical perception [5]. Though previous research suggests that such a model can be built, previous research has not provided an empirically validated model of the lowlevel perception, decision, and motor processes that people use when they look for a known item in a menu or on a computer screen $[6,7]$. By providing a model of the lowlevel cognitive processing involved in these visual search tasks, this dissertation will break new ground.

\section{COGNITIVE MODELING}

Models of human performance permit aspects of user interfaces to be evaluated for usability by making predictions based on task analysis and established principles of human performance [1, 3]. As well, when accurately predicting performance times recorded with human subjects, cognitive modeling can reveal the low-level perception, decision, and motor processes that people use to accomplish specific tasks [4]. In my dissertation work, I will build and run models using the EPIC (Executive Process Interactive Control) cognitive architecture [4]. EPIC provides a general framework for simulating a human interacting with their environment to accomplish a task, and is well-suited to model visual search tasks. EPIC resembles the Model Human Processor [1], but differs in that EPIC is a precise computational model, has a programmable production-rule cognitive processor, and incorporates more specific constraints synthesized from human performance literature.

\section{PRELIMINARY INVESTIGATION}

As a preliminary investigation for this dissertation, Hornof \& Kieras [2] built cognitive models to investigate how people search for a known target item in a randomly ordered pull-down menu. In these models, we varied the strategy that the model employed (serial versus parallel processing of menu items, and random versus systematic search) and one parameter in the task environment (the number of menu items fitting into the fovea simultaneously). Varying these conditions, we constructed models using the EPIC architecture and ran the models. We compared the selection times predicted by the models with selection times of 
human subjects performing the same menu task. Comparing the predicted and observed times, the model that best fits the empirical data indicates that (1) people process more than one menu item at a time, and (2) people search menus using both random and systematic search strategies. This preliminary investigation, which is discussed more extensively in [2], demonstrates the usefulness and plausibility of the project proposed here.

\section{RESEARCH PLAN}

Key components of the research plan for this dissertation include modeling additional menu data, extracting key elements of search strategies from the menu models to build a general search model, and using this general search model to explain some screen layout design guidelines.

\section{Model additional menu data.}

As a preliminary investigation, we successfully modeled data of subjects using randomly ordered menus. A critical next step is to model data from the same experiment, but from subjects using sorted menus. The strategies from these two search tasks - randomly ordered menus and sorted menus - should provide a basis for understanding search strategies that people use for more general search tasks. In more general search tasks, people will either (1) have no idea where the target will be (as in randomly ordered menus), or (2) have an idea of where the target will be (as in ordered menus). The menu models should thus help to guide the next step, which will be to build a more general model of visual search.

Extract key elements of search strategies and build a general search model.

After figuring out plausible strategies that people use for visual search in menu selection tasks, I intend to generalize these search strategies to more general search tasks. I intend to build models of these more general search tasks, and to validate these models with empirical data. Ideally, these more general search tasks will correspond closely to visual search problems in real-world tasks, such as viewing pages on the world wide web.

\section{Explain screen layout design guidelines.}

The search strategies used in the general search models could be used, as part of the dissertation work, to explain how screen layout design guidelines affect the cognitive processes involved in visual search tasks. The plan would be to (1) identify a few screen layout design guidelines that should help people search screen layouts more efficiently, (2) find specific instances in real-world interfaces (such as on the world wide web) in which these guidelines are adhered to and violated, (3) build cognitive models that use the hypothesized general search strategies to predict how a human would accomplish these real-world visual search tasks, and (4) validate these predictions with data collected from humans accomplishing the same tasks. As a result of this effort, I should be able to explain how some screen layout design guidelines ultimately help people find things on a computer screen, and how other guidelines might not actually aid visual search.

\section{SIGNIFICANCE}

This dissertation should provide the field of humancomputer interaction with an empirically validated model of the low-level perception, decision, and motor processes that people use when they look for an item on a computer screen. The significance of this contribution includes: (1) The project will increase our understanding of human cognition by helping to explain how people conduct visual searches, what processes are involved, and how the processes interact. (2) The project will help to guide future research in this domain by helping to determine what is a useful experimental paradigm, and what data still need to be collected. (3) The project will help interface designers by explaining how people conduct visual searches, by explaining how some guidelines expedite low-level cognitive processes, and by providing theory needed to build a software tool that would automatically predict search times based on a description of the visual task and screen layout.

\section{ACKNOWLEDGMENTS}

This work is supported by the Advanced Research Projects Agency under order number B328, monitored by NCCOSC under contract number N66001-94-C-6036 awarded to David Kieras.

\section{REFERENCES}

1. Card, S. K., Moran, T. P., \& Newell, A. (1983). The Psychology of Human-Computer Interaction. Hillsdale, NJ: Lawrence Erlbaum Associates.

2. Hornof, A. J., \& Kieras, D. E. (1997). Cognitive Modeling Reveals Menu Search is Both Random and Systematic. Proceedings of CHI '97, New York: ACM, 107-114.

3. John, B. E., \& Kieras, D. E. (1994). The GOMS family of analysis techniques: Tools for design and evaluation. (Technical Report No. CMU-CS-94-181) Carnegie Mellon University School of Computer Science.

4. Kieras, D. E., \& Meyer, D. E. (1997). An overview of the EPIC architecture for cognition and performance with application to human-computer interaction. Human-Computer Interaction, 12(4), 391-438.

5. Lohse, G. L. (1993). A cognitive model for understanding graphical perception. Human-Computer Interaction, 8, 353-388.

6. Paap, K. R., \& Roske-Hofstrand, R. J. (1988). Design of menus, Handbook of Human Computer Interaction. Amsterdam: Elsevier Science Publishers, 205-235.

7. Scott, D. (1993). Visual search in modern humancomputer interfaces. Behaviour and Information Technology, 12(3), 174-189.

8. Sears, A. (1995). AIDE: A step toward metric-based interface development tools. Proceedings of the ACM Symposium on User Interface Software and Technology, UIST '95, 101-110.

9. Tullis, T. S. (1988). A System for Evaluating Screen Formats: Research and Application. In R. Hartson \& D. Hix (Eds.), Advances in Human-Computer Interaction. (Vol. 2). Norwood, NJ: Ablex, 214-286. 